\title{
Direct estimate of Hubble's constant from the double quasar
}

\section{from C. Martin Gaskell}

DETERMINATION of the time delay between observed variations in the two images of the first gravitationally lensed quasar to be discovered $(0957+561$ or 'the double quasar') has enabled Hubble's constant $\left(H_{\mathrm{o}}\right)$ to be estimated directly ${ }^{1}$. This way of measuring $H_{\mathrm{o}}$ short-circuits the many intermediate steps in 'classical' estimations but, unfortunately, is very model dependent.

The double quasar was discovered by D. Walsh, R.F. Carswell and R.J. Weymann as a pair of quasars, 6 arcseconds apart, with virtually identical spectra ${ }^{2}$. Subsequent research verified their initial guess that these two images come from one and the same quasar: the gravity of a galaxy in the line of observation is bending the light and producing two images of the more distant quasar ${ }^{3}$. Long before this system was discovered it had been pointed out that variability of a gravitationally lensed object would allow an estimate of the distances involved and hence of $H_{0}$, the constant that relates the velocity of recession of the galaxy or quasar to its distance $\left(1 / H_{0}\right.$ is the approximate age of the Universe $)^{4}$. In essence, the method is simple: if the shape of the various light paths is known, then the time delay due to the difference in light travel time) between seeing a given variation in one image and the other, gives the scale size. (For the double quasar there are actually three images, but the third is very faint.)

Like virtually all quasars ${ }^{5}, 0957+561$ was quickly shown to exhibit 'flickerings' in brightness (of 30 per cent or so) that last weeks or months ${ }^{6}$. Once that was known, a number of observatories mounted photometric monitoring programmes, and, while waiting for the results, theoreticians refined the model of the mass distribution in the 'lensing' galaxy and the cluster in which it is located ${ }^{7,8}$. The expectation was that variability of the northern image would precede variability of the southern image by a couple of years.

R. Florentin-Nielsen and K. Augustesen of Copenhagen University Observatory were among the observers following $0957+561$. They took repeated photographs with a 20 -inch Schmidt camera, a small telescope by modern standards, and in much poorer sky conditions than United States' observers, but with the advantage of being far enough north to follow the double quasar all year round. In early 1980 , the southern image brightened while the northern one stayed constant; the southern image was presumably showing some event that could have been seen a year or two earlier in the northern image. Both images then stayed constant in brightness until
1982 when the northern image started to brighten by 30 per cent while the southern image stayed constant. In 1983, the northern image started to fade. There had been a clear 'event'. It was then just a matter of waiting until the southern image followed suit for an estimate of $H_{0}$ to be made. By July 1984 it was clear to Florentin-Nielsen that the long-awaited rise in the southern image had begun. In his recent paper ${ }^{1}$, he estimates the time delay to be $1.55 \pm 0.1$ years and derives $H_{0}$ as $77 \mathrm{~km} \mathrm{~s}^{-1} \mathrm{Mpc}^{-1}$ corresponding to a Hubble age $\left(1 / H_{\mathrm{o}}\right)$ of $13 \times 10^{9}$ years.

How reliable is this estimate of $H_{0}$ ? The formal observational error is only $\pm 5 \mathrm{~km}$ $\mathrm{s}^{1} \mathrm{Mpc}^{-1}$. Classical astronomical methods (for example, using Cepheid variable stars) currently yield $H_{\mathrm{o}}$ values of $43-95 \mathrm{~km} \mathrm{~s}^{-1}$ $\mathrm{Mpc}^{-1}$, and a recent review favours 68 or 82 $\mathrm{km} \mathrm{s}^{-1} \mathrm{Mpc}^{-1}$, depending on the methods used. The latest work on type I supernovae ${ }^{10}$ favours a value of $58 \mathrm{~km} \mathrm{~s}^{-1}$ $\mathrm{Mpc}^{-1}$. The Florentin-Neilsen value is clearly well within the range under debate.

Within a year or two the observational uncertainty in the time lag should have been narrowed down by a factor of two or more. But the real uncertainty is greater. To calculate $H_{0}$, Florentin-Nielsen relies on detailed modelling by Borgeest and Refsdal ${ }^{8}$ of the mass distribution in the lensing galaxy and the cluster that contains it. The question is whether Borgeest and Refsdal have correctly modelled the influence of the cluster on the estimate of $H_{0}$. They believe it is less influenced by the cluster parameters than had been feared by, for example, Dyer and Roeder ${ }^{7}$, and that the effect of the cluster galaxies might be leading to a slight overestimate of $H_{0}$ from the double quasar. Nevertheless, it remains the largest source of error.

Fortunately, the free parameters in the mass distributions may soon be narrowed down by radio observations of very high spatial resolution that are underway. The models of the mass distributions will have to match the twisting and distortion seen in the radio structure. If they do, then there is some confidence that the time delays, and hence $H_{\mathrm{o}}$, have been calculated correctly. Meanwhile, optical observers will be calmly continuing to follow the two images to ensure that the current outburst in the southern image really matches the $1982-3$ record for the northern image. If not, some major rethinking will be necessary.

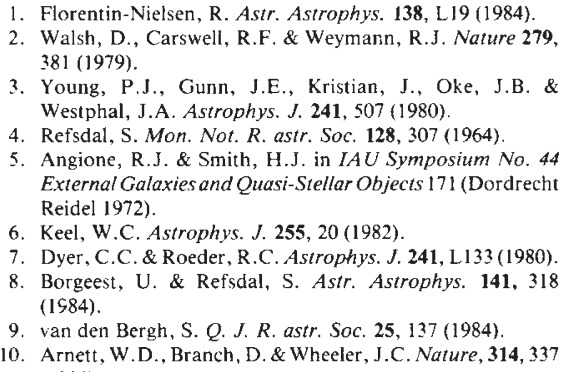

C.Martin Gaskell is in the Astronomy Department, University of Texas, Austin, Texas 78712, USA.

\section{Parasitology}

\section{Towards schistosomiasis vaccines}

from F.E.G. Cox

SCHISTOSOMIASIS, one of the world's major diseases, is caused by adult worms that inhabit blood vessels associated with the lower intestine or bladder. The worms survive in these immunologically exposed sites largely because while migrating there as juveniles they acquire host-like surface antigens from the skin, where they enter the body. So effective is this disguise that it is not at all clear if there is any effective immunity in man ${ }^{1}$. Vaccines consisting of irradiated juvenile worms ${ }^{2}$ have been the basis of successful field trials in cattle in the Sudan ${ }^{3}$ and China ${ }^{4}$ but are unlikely to be acceptable for human use. Therefore, several groups have investigated the potential of extracts of juvenile and adult worms. The results are disappointing, although some purified surface antigens have been used with limited success in mice and rats. The most recent study indicates that some protection can be induced in monkeys 5 . This is a major step forward.
Maire Smith and John Clegg, of the Wellcome Research Laboratories, have identified and purified two surface antigens associated with both juvenile and adult worms ${ }^{5}$. The larger molecule, given twice with alhydrogel adjuvant, induces partial protection both in mice and cynomolgus monkeys. The smaller antigen, which could be extracted from the adult worms only with difficulty, also protects mice. These results are promising in that antigens common to the surfaces of both juvenile and adult worms have some protective capacity, but disappointing in that the levels of protection are low and inconsistent. Nevertheless, they indicate that the approach of identifying and purifying surface antigens is worth pursuing. The next step is to clone the genes encoding these molecules, to express them and to test the products.

Although it is now possible to predict future directions of research, it would be 